\title{
Draft genome sequences of four Achromobacter ruhlandii strains isolated from cystic fibrosis patients
}

\author{
Elenice RA Rodrigues' ${ }^{1}$ Géssica A Rocha', Alex G Ferreira', \\ Robson S Leão', Rodolpho M Albano ${ }^{2}$, Elizabeth A Marques ${ }^{1 /+}$ \\ ${ }^{1}$ Universidade do Estado do Rio de Janeiro, Faculdade de Ciências Médicas, Departamento de Microbiologia, Imunologia e Parasitologia, \\ Rio de Janeiro, RJ, Brasil ${ }^{2}$ Universidade do Estado do Rio de Janeiro, Instituto de Biologia Roberto Alcântara Gomes, \\ Departamento de Bioquímica, Rio de Janeiro, RJ, Brasil
}

Achromobacter species are being increasingly isolated from the respiratory tract of cystic fibrosis patients. Recent reports indicate that Achromobacter ruhlandii is a potential human pathogen in cystic fibrosis-related infections. Here we report the draft genome of four A. ruhlandii strains isolated from cystic fibrosis patients in Brazil. This report describes A. ruhlandii as a potential opportunistic pathogen in cystic fibrosis and provides a framework to for additional enquires into potential virulence factors and resistance mechanisms within this species.

Key words: Achromobacter ruhlandii - genome sequence - cystic fibrosis

Achromobacter ruhlandii is a Gram-negative bacterium naturally found in soil (Packer \& Vishniac 1955). However, recent reports indicate that $A$. ruhlandii is a potential human pathogen in cystic fibrosis-related infections (Ridderberg et al. 2012, Spilker et al. 2012a). A PAN-resistant Achromobacter clone, designated the danish epidemic strain (DES), causing infection in cystic fibrosis patients in Copenhagen (Hansen et al. 2006) and Aarhus (Ridderberg et al. 2011), was recently identified by multilocus sequence typing (MLST) as $A$. ruhlandii (Ridderberg et al. 2012). A. ruhlandii has also been reported as the second most commonly isolated Achromobacter species from cystic fibrosis patients (Spilker et al. 2012b).

Here we describe draft genome sequences of four A. ruhlandii strains isolated from sputum of Brazilian cystic fibrosis patients attended at Instituto Nacional da Saúde da Mulher, da Criança e do Adolescente Fernandes Figueira (IFF-FIOCRUZ) and Hospital Universitário Pedro Ernesto (HUPE-UERJ), in 2007 and 2008. The isolates were identified to species level by sequencing seven housekeeping genes that were subsequently submitted to the Achromobacter MLST database where they were assigned to specific STs (Spilker at al. 2012a; http://pubmlst.org/achromobacter/). Furthermore, the species specific marker genes for $A$. xylosoxidans (blaOXA-114 $)$ and for $A$. ruhlandii (bla ${ }_{\mathrm{OXA}-258}$ ) were amplified and sequenced to confirm species assignment (Turton et al. 2011, Papalia et al. 2013). Accordingly, the sequences from all four strains showed identity with $b l_{\text {oxa-258 }}$.

doi: 10.1590/0074-02760160130

Financial support: FAPERJ (E-26/110.742/2013), CNPq

(471480/2012-6).

+ Corresponding author: marbe@uerj.br

Received 30 May 2016

Accepted 31 August 2016
Strains 6241, 7863, 7022 and 8173 were assigned STs $35,204,36$ and 35, respectively. ST 35 was the only one shared between the two study centers. Minimal inhibitory concentration against ceftazidime, ciprofloxacin, imipenem and trimetoprim/sulphametoxazol was determined with the E-test strip (AB Biodisk, Solna, Sweden). The four samples were susceptible to antibiotics with the exception of strain 7022 that was resistant to trimethoprim/sulfamethoxazole.

Genomic libraries were constructed by transposon tagmentation with the Nextera XT DNA Library Prep kit (Illumina Inc, USA). Sequencing was performed for each isolate with the 500 cycle MiSeq Reagent v2 kit on a MiSeq benchtop instrument (Illumina). Paired-end sequence reads obtained for each of the isolates ranged from 2,017,226 to 3,232,222. Reads were corrected and assembled de novo into scaffolds with Spades 3.5 genome assembler (Bankevich et al. 2012). The Rapid Annotation using System Technology (RAST) v.2.0 server (http://rast.nmpdr.org) was used for general genome annotation and the following databases were used to refine RAST results: PHAge search tool (PHAST) (http:// phast.wishartlab.com/), IS Blast Server (IS FINDER) (https://www-is.biotoul.fr/) and Antibiotic Resistance Genes Database-ARDB (http://ardb.cbcb.umd.edu/). The resulting scaffolds per isolate ranged from 89-111 with an average genome size of 6,481,38 bp (ranging from $6,289,667$ to $6,686,778$ ) and 56 or 58 RNA genes. The results of these analyses are summarised on Table I along with their GenBank accession numbers.

The four $A$. ruhlandii strains were compared with the genome of $A$. xylosoxidans NH-44784-1996 (Jakobsen et al. 2013), an isolate from a cystic fibrosis patient. The genes involved in pathogenicity were identified, according to the annotation obtained in the RAST server and are summarised on Table II.

Genes responsible for resistance to antibiotics (marC, macA macB, mexI, mexD, mexA, mexB, OprM, mexX, cmeA, cmeB, cmeC, bla OXA258 $\left._{1}\right)$ were annotated, however, only strain 7022 showed the presence of SHV- 
TABLE I

Overview of genome sequence assemblies

\begin{tabular}{lcccccc}
\hline Strain & Hospital & $\begin{array}{c}\text { Total of reads } \\
\left(\mathrm{n}^{\circ}\right)\end{array}$ & $\begin{array}{c}\text { Contigs } \\
\left(\mathrm{n}^{\circ}\right)\end{array}$ & $\begin{array}{c}\text { Genome size } \\
(\mathrm{bp})\end{array}$ & $\begin{array}{c}\text { RNA genes } \\
\left(\mathrm{n}^{\circ}\right)\end{array}$ & $\begin{array}{c}\text { Accession } \\
\left(\mathrm{n}^{\circ}\right)\end{array}$ \\
\hline 6241 (ST 35) & IFF-FIOCRUZ & $2,017,226$ & 91 & $6,686,778$ & 58 & LVKM00000000 \\
7863 (ST 204) & HUPE-UERJ & $2,849,474$ & 111 & $6,450,125$ & 56 & LVKO00000000 \\
7022 (ST 36) & IFF-FIOCRUZ & $3,232,222$ & 89 & $6,498,950$ & 56 & LVKN00000000 \\
8173 (ST 35) & HUPE-UERJ & $2,550,870$ & 90 & $6,289,667$ & 58 & LVKP00000000 \\
\hline
\end{tabular}

HUPE-UERJ: Hospital Universitário Pedro Ernesto - Universidade do Estado do Rio de Janeiro; IFF-FIOCRUZ: Instituto Nacional da Saúde da Mulher, da Criança e do Adolescente Fernandes Figueira - Fundação Oswaldo Cruz.

TABLE II

Identified genes in Achromobacter ruhlandii involved in pathogenicity

\begin{tabular}{|c|c|c|c|c|c|c|}
\hline Product & Gene name & $\begin{array}{l}\text { A. xylosoxidans } \\
\text { NH44784-1996 }\end{array}$ & $\begin{array}{l}\text { AR } \\
6241\end{array}$ & $\begin{array}{c}\text { AR } \\
7022\end{array}$ & $\begin{array}{c}\text { AR } \\
7863\end{array}$ & $\begin{array}{c}\text { AR } \\
8173\end{array}$ \\
\hline \multicolumn{7}{|l|}{ Type II } \\
\hline General secretion pathway & Type C,D,E,F,G,H,I,J,K,L,M,N & + & + & + & + & + \\
\hline \multicolumn{7}{|l|}{ Type III } \\
\hline Outer membrane pore forming protein & YscC,MxiD,HrcC, InvG & + & + & + & + & + \\
\hline Inner membrane protein & YscU,SpaS,EscU,HrcU,SsaU & + & + & + & + & + \\
\hline Inner membrane protein & YscT,HrcT,SpaR,EscT,EpaR1 & + & + & + & + & + \\
\hline Inner membrane protein & YscS & + & + & + & + & + \\
\hline Inner membrane protein & YscR,SpaR,HrcR,EscR & + & + & + & + & + \\
\hline Inner membrane protein & YscQ & + & + & + & + & + \\
\hline Spans bacterial envelope protein & $\mathrm{YscO}$ & + & - & - & - & - \\
\hline Cytoplasmic protein & YscL & + & + & + & + & + \\
\hline Putative type III secretion protein & - & + & - & - & - & - \\
\hline Bridge between inner and outer membrane lipoprotein & YscJ,HrcJ,EscJ, PscJ & + & + & + & + & + \\
\hline Chaperone protein for YopD & SycD & + & + & + & + & + \\
\hline Cytoplasmic LcrG inhibitor & $\mathrm{LcrV}$ & + & - & - & - & - \\
\hline Inner membrane channel protein & LcrD,HrcV,EscV,SsaV & + & + & + & + & + \\
\hline \multicolumn{7}{|l|}{ Type VI } \\
\hline ClpB protein & ClpB & + & + & + & + & + \\
\hline IcmF-related protein & IcmF & + & + & + & + & + \\
\hline Protein ImpG/VasA & ImpG & + & + & + & + & + \\
\hline Sigma-54 dependent transcriptional regulator & - & + & + & + & + & + \\
\hline Uncharacterized protein ImpA & $\operatorname{ImpA}$ & + & + & + & + & + \\
\hline Uncharacterized protein ImpB & $\operatorname{ImpB}$ & + & + & + & + & + \\
\hline Uncharacterized protein ImpC & ImpC & + & + & + & + & + \\
\hline Uncharacterized protein ImpD & $\operatorname{ImpD}$ & + & + & + & + & + \\
\hline Uncharacterized protein ImpF & $\mathrm{ImpF}$ & + & + & + & + & + \\
\hline Uncharacterized protein ImpH/VasB & $\mathrm{ImpH}$ & + & + & + & + & + \\
\hline Uncharacterized protein ImpJ/VasE & ImpJ & + & + & + & + & + \\
\hline VgrG protein & VgrG & + & - & - & - & - \\
\hline \multicolumn{7}{|l|}{ Type VII } \\
\hline Sigma-fimbriae chaperone protein & - & + & + & + & + & + \\
\hline Sigma-fimbriae tip adhesin & - & + & + & + & + & + \\
\hline Sigma-fimbriae usher protein & - & + & + & + & + & + \\
\hline \multicolumn{7}{|l|}{ Adhesion } \\
\hline PGA outer membrane secretin & PgaA & + & + & + & + & + \\
\hline PGA synthesis deacetylase & PgaB & + & + & + & + & + \\
\hline PGA synthesis N-glycosyltransferase & $\mathrm{PgaC}$ & + & + & + & + & + \\
\hline PGA synthesis auxiliary protein & PgaD & + & + & + & + & + \\
\hline
\end{tabular}

-: refers to the ausence of gene; +: refers to the presence of gene; AR: A. ruhlandii. 
TABLE III

Intact phages and incomplete prophages regions identified in Achromobacter ruhlandii strains

\begin{tabular}{|c|c|c|c|c|}
\hline \multirow{2}{*}{$\begin{array}{l}\text { Phages / incomplete prophage regions } \\
\text { Intact phages }\end{array}$} & \multicolumn{4}{|c|}{ Strain } \\
\hline & 6241 & 7022 & 7863 & 8173 \\
\hline PHAGE-Burkho-phi644-2-NC-009235 & + & + & + & + \\
\hline PHAGE-Burkho-KS14-NC-015273 & - & + & - & - \\
\hline PHAGE-Erwini-phiEt88-NC-015295 & - & - & + & - \\
\hline PHAGE-Pseudo-YMC11/02/R656-NC-028657 & - & - & - & + \\
\hline PHAGE-Burkho-Bcep176-NC-007497 & - & - & - & + \\
\hline \multicolumn{5}{|l|}{ Incomplete prophage regions } \\
\hline PHAGE-Salmon-SEN34-NC-028699 & + & + & + & + \\
\hline PHAGE-Burkho-BcepB1A-NC-005886 & - & + & - & - \\
\hline PHAGE-Burkho-BcepC6B-NC-005887 & - & + & - & - \\
\hline PHAGE-Entero-fiAA91-ss-NC-022750 & - & + & - & - \\
\hline PHAGE-Yellow-1-NC-028112 & - & - & + & - \\
\hline
\end{tabular}

$+:$ refers to the presence of these intact phages or incomplete prophages regions in strains; -: refers to the absence of intact phages or incomplete prophage regions in strains.

5a and APH(3')-II. Furthermore, we also observed two resistance genes that are usually associated with mobile elements, sull and dfra26. However, in these genomes they could be not associated with these elements, being randomly located in the chromosome (Antunes et al. 2004, Miranda et al. 2004, Garza-Ramos \& Romero 2007, Grape et al. 2007). A comparison of our A. ruhlandii samples with other genomic sequences of different species found in the databases demonstrated the presence of IS and transposable elements that were related to ISBcen18 (Burkholderia cenocepacia J2315), ISPa43 (Pseudomonas aeruginosa), TnAs2 (Aeromonas salmonicida), TnAs3 (Aeromonas salmonicida subsp. salmonicida A449 plasmid 4), ISRme12 (Ralstonia metallidurans CH34), ISBmu5 (Burkholderia multivorans ATCC 17616), ISBcen10 (Burkholderia cenocepacia J2315), ISStma15 (Stenotrophomonas maltophilia K279a), ISPst3 (Pseudomonas stutzeri OM1), IS408 (Burkholderia cenocepacia ATCC17616),ISPa38 (Pseudomonas aeruginosa DK2), ISPa39 (Pseudomonas aeruginosa DK2), ISPa40 (Pseudomonas aeruginosa DK2), ISBcen23 (Burkholderia cenocepacia HI2424), IS1474 (Pseudomonas alcaligenes ATCC14094 / Pseudomonas alcaligenes NCIB9867 P25X / Pseudomonas putida NCIB9869 P35X) and IS1162 (Pseudomonas fluorescens $\mathrm{ST}$ plasmid $\mathrm{pEG}$ ). This illustrates the potential ability of $A$. ruhlandii to carry genetic and transferable elements that could contribute to the dissemination/acquisition of antimicrobial resistance mechanisms.

Five intact phages (PHAGE-Burkho-phi6442-NC-009235, PHAGE-Burkho-KS14-NC-015273, PHAGE-Erwini-phiEt88-NC-015295, PHAGE-Pseudo-YMC11/02/R656-NC-028657 and PHAGE-Burkho-Bcep176-NC-007497) and five incomplete prophage regions (PHAGE-Salmon-SEN34-NC-028699, PHAGE-Burkho-BcepB1A-NC-005886, PHAGE-Burkho-BcepC6B-NC-005887,PHAGE-Entero-fiAA91-ssNC-022750, PHAGE-Yellow-1-NC-028112) were also detected in our A. ruhlandii strains (Table III).
This whole Genome Shotgun project has been deposited at DDBJ/ENA/GenBankunder the accession LVKM00000000, LVKO00000000, LVKN00000000 and LVKP00000000. The version described in this paper is version LVKM01000000, LVKO01000000, LVKN01000000 and LVKP01000000.

\section{REFERENCES}

Antunes P, Machado J, Sousa JC, Peixe L. Dissemination of sulfonamide resistance genes (sull, sul2, and sul3) in Portuguese Salmonella enterica strains and relation with integrons. Antimicrob Agents Chemother. 2004; 49(2): 836-9.

Bankevich A, Nurk S, Antipov D, Gurevich AA, Dvorkin M, Kulikov AS, et al. SPAdes: a new genome assembly algorithm and its applications to single-cell sequencing. J Comput Biol. 2012; 19(5): 455-77.

Garza-Ramos U, Romero EM. SHV-type extended-spectrum B-lactamase (ESBL) are encoded in related plasmids from enterobacteria clinical isolates from Mexico. Salud Publica Mex. 2007; 49(6): 415-21.

Grape M, Sundström L, Kronvall G. Two new $d f r$ genes in trimethoprim-resistant integron-negative Escherichia coli isolates. Antimicrob Agents Chemother. 2007; 51(5): 1863-4.

Hansen RC, Pressler T, Høiby N, Gormsen M. Chronic infection with Achromobacter xylosoxidans in cystic fibrosis patients; a retrospective case control study. J Cyst Fibros. 2006; 5(4): 245-51.

Jakobsen TH, Hansen MA, Jensen PØ, Hansen L, Riber L, Cockburn $\mathrm{A}$, et al. Complete genome sequence of the cystic fibrosis pathogen Achromobacter xylosoxidans NH44784-1996 complies with important pathogenic phenotypes. PLoS ONE. 2013; 8(7): e68484.

Miranda G, Castro N, Leaños B, Valenzuela A, Garza-Ramos U, Rojas T, et al. Clonal and horizontal dissemination of Klebsiella pneumonia expressing SHV-5 extended-spectrum ß-lactamase in a Mexican Pediatric Hospital. J Clin Microbiol. 2004; 42(1): 30-5.

Packer L, Vishniac W. Chemosynthetic fixation of carbon dioxide and characteristics of hydrogenase in resting cell suspensions of $\mathrm{Hy}$ drogenomonas ruhlandii nov. spec. J. Bacteriol. 1955; 70(2): 216-23.

Papalia M, Almuzara M, Cejas D, Traglia G, Ramírez MS, Galanternik L, et al. OXA-258 from Achromobacter ruhlandii: a species-specific marker. J Clin Microbiol. 2013; 51(5): 1602-5. 
Ridderberg W, Bendstrup KE, Olesen HV, Jensen-Fangel S, NørskovLauritsen N. Marked increase in incidence of Achromobacter xylosoxidans infections caused by sporadic acquisition from the environment. J Cyst Fibros. 2011; 10(6): 466-9.

Ridderberg W, Wang M, Nørskov-Lauritsen N. Multilocus sequence analysis of isolates of Achromobacter from patients with cystic fibrosis reveals infecting species other than Achromobacter $x y$ losoxidans. J Clin Microbiol. 2012; 50(8): 2688-94.

Spilker T, Vandamme P, LiPuma JJ. Identification and distribution of
Achromobacter species in cystic fibrosis. J Cyst Fibros. 2012b; 12(3): 298-301.

Spilker T, Vandamme P, LiPuma JJ. Multilocus sequence typing scheme infers population structure and reveals several putative novel Achromobacter species. J Clin Microbiol. 2012a; 50(9): 3010-5.

Turton JF, Mustafa N, Shah J, Hampton CV, Pike R, Kenna DT. Identification of Achromobacter xylosoxidans by detection of the bla $_{\text {on }}$ gene intrinsic in this species. Diagn Microbiol Infect Dis. 2011; 70(3): 408-11 